\title{
Diseño Integrado para Viviendas de Alto Desempeño (Casa+)
}

\author{
Integrated Design for High Performance Housing (Casa+)
}

\author{
Rodrigo García Alvarado \\ Universidad del Bío-Bío, Chile \\ rgarcia@ubiobio.cl \\ Flavio Celis D’Amico \\ Universidad de Alcalá de Henares, España \\ celis.flavio@gmail.com \\ Ernesto Echeverria Valiente \\ Universidad de Alcalá de Henares, España \\ echevus@gmail.com
}

\author{
Maureen Trebilcock Kelly \\ Universidad del Bío-Bío, Chile \\ mtrebilcock@ubiobio.cl \\ Muriel Diaz \\ Universidad del Bío-Bío, Chile \\ miauriel@gmail.com \\ Gerth Wandersleben \\ Universidad del Bío-Bío, Chile \\ gerthwwm@gmail.com
}

\begin{abstract}
The growing environmental constraints encourage new strategies for housing projects. This work exposes the application of energy simulations and integrated design to develop an innovative prefabricated housing system called "Casa +" (House Plus), carried out by an international research team with industrial collaboration. The proposal achieves high density, flexible growth, environmental comfort and minimum fuel consumption, for demonstrate the use of new analysis technologies and design methods to substantially improve residential quality.
\end{abstract}

Keywords: Integrated design, High-performance buildings, Prefabricated construction; Housing; energy simulations.

\section{Introducción}

La mitad de los recursos mundiales, del agua y la energía consumida, se destina a las construcciones, provocando gran parte del calentamiento global (IEA, 2012). Debido a que los combustibles utilizados en la fabricación de materiales y funcionamiento de los edificios provienen en su mayoría de fuentes carbonizadas no-renovables y foráneas, especialmente en Chile (CNE, 2009). A la vez que se mantienen bajas condiciones ambientales, con relevantes diferencias económicas (CDT, 2010). En particular en las viviendas, que alojan la mayor parte de las actividades humanas y requieren un proceso permanente de construcción, por el crecimiento urbano y déficit habitacional.

La zona centro-sur de Chile, que posee un clima templado estacional, presenta un amplio predominio de viviendas aisladas/pareadas ampliadas, con débiles condiciones ambientales y demandas energéticas relevantes, en especial de calefacción (Celis et al, 2012). Además de un desarrollo urbano extendido y socialmente segregado. Se han planteado varias consideraciones para mejorar el desempeño de las viviendas en esta zona, como la mayor aislación de las envolventes y compacidad de los volúmenes (Bustamante, 2009), lo que contribuye también a la densidad urbana e integración social. Reduciendo el transporte y extensión de las ciudades, para mitigar el impacto ambiental e incrementar la calidad de vida de las personas.
Sin embargo, escasamente se han incorporado estos aspectos en las regulaciones y prácticas profesionales, y las viviendas siguen ejecutándose con debilidades ambientales. Actualmente se dispone de nuevas tecnologías de proyecto, análisis y construcción, así como estrategias de diseño integrado que han permitido formular de edificios con alto desempeño ambiental (García et al, 2012). Por esta razón, en el marco de un proyecto de investigación internacional, con la colaboración de empresas locales, se aplicaron estas posibilidades en una propuesta de construcción residencial industrializada para la zona, denominada "Casat". Esta iniciativa asumió como objetivo revisar nuevas consideraciones y procesos de diseño integrado en viviendas unifamiliares para el centro-sur de Chile, que permitan lograr densidad y crecimiento flexible, con un alto desempeño ambiental y mínimo consumo energético.

\section{Diseño Integrado}

El proyecto aplicó tres aspectos claves de trabajo colabora-tivo para edificios de alto desempeño:

1. Definición previa y consensuada de metas prestacionales del diseño.

2. Utilización de herramientas de evaluación para definir la volumetría, características constructivas y urbanas del proyecto.

3. Reuniones multidisciplinarias regulares para desarrollar y revisar el proyecto. 
El equipo de trabajo estuvo encabezado por uno de los investigadores extranjeros con experiencia proyectual (Dr. Flavio Celis), convocando profesionales de distintas especialidades y roles (Tabla 1). Incluyendo representantes de una constructora e inmobiliaria local, con interés de aplicar el sistema y trayectoria en desarrollos residenciales. Se realizaron diversas sesiones (Tabla 2), con distribución de tareas, compartiendo resultados y discutiendo diferentes condiciones que permitieron refinar sucesivamente el diseño.

Tabla 1: Lista de Participantes

\begin{tabular}{|l|l|l|}
\hline Profesión & Rol & Dedicación \\
\hline Arquitecto Dr. & Coordinador & Alta \\
\hline Arquitecto Dr. & Supervisor & Baja \\
\hline Arquitecto Dr. & Asesor Bio-climático & Baja \\
\hline Arquitecto Ms.(c) & Simulaciones Ambientales & Media \\
\hline Arquitecto Ms.(c) & Modelación y Diseño BIM & Media \\
\hline Ing. Civil & Cálculo Estructural & Baja \\
\hline Ing. Constructor & Presupuestos & Baja \\
\hline Ing. Mecánico Ms. & Climatización & Baja \\
\hline Arquitecto & Representante Mandante & Media \\
\hline Ing. Constructor & Representante Ejecución & Baja \\
\hline
\end{tabular}

Tabla 2: Programa de Reuniones

\begin{tabular}{|c|c|c|}
\hline Sesión & Actividades Principales & Participación \\
\hline $1-$ & $\begin{array}{l}\text { Definición del problema, de los } \\
\text { intervinientes y metas de prestación. }\end{array}$ & Alta \\
\hline $2-$ & $\begin{array}{l}\text { Integración del mandante y el } \\
\text { constructor al equipo de DI. }\end{array}$ & Baja \\
\hline $3-$ & $\begin{array}{l}\text { Prediseño en base a objetivos y metas } \\
\text { acordados en } 1,2 \text { y } 3 .\end{array}$ & Media \\
\hline 4- & $\begin{array}{l}\text { Incorporación de ingeniero de clima y } \\
\text { de construcción, bases de presupuesto. }\end{array}$ & Media \\
\hline $5-$ & $\begin{array}{l}\text { Revisión de la Modelación BIM del } \\
\text { Sistema Prefabricado y Plantas } \\
\text { Arquitectónicas }\end{array}$ & Media \\
\hline $6-$ & $\begin{array}{l}\text { Formulación de Condiciones de } \\
\text { Simulación Ambiental }\end{array}$ & Baja \\
\hline $7-$ & $\begin{array}{l}\text { Revisión del Presupuesto y } \\
\text { Especificación Constructiva }\end{array}$ & Baja \\
\hline $8-$ & $\begin{array}{l}\text { Análisis de resultados de Simulación } \\
\text { Ambiental y Diseño de Unidades }\end{array}$ & Media \\
\hline 9- & $\begin{array}{l}\text { Revisión de la Modelación BIM de } \\
\text { Conjuntos Urbanos y Láminas. }\end{array}$ & Alta \\
\hline $10-$ & $\begin{array}{l}\text { Propuestas de Climatización, } \\
\text { Iluminación Natural y Energía Solar }\end{array}$ & Baja \\
\hline $11-$ & $\begin{array}{l}\text { Revisión crítica integrada de los } \\
\text { resultados obtenidos. }\end{array}$ & Media \\
\hline $12-$ & $\begin{array}{l}\text { Reunión final y presentación del } \\
\text { sistema y propuestas habitacionales }\end{array}$ & Media \\
\hline
\end{tabular}

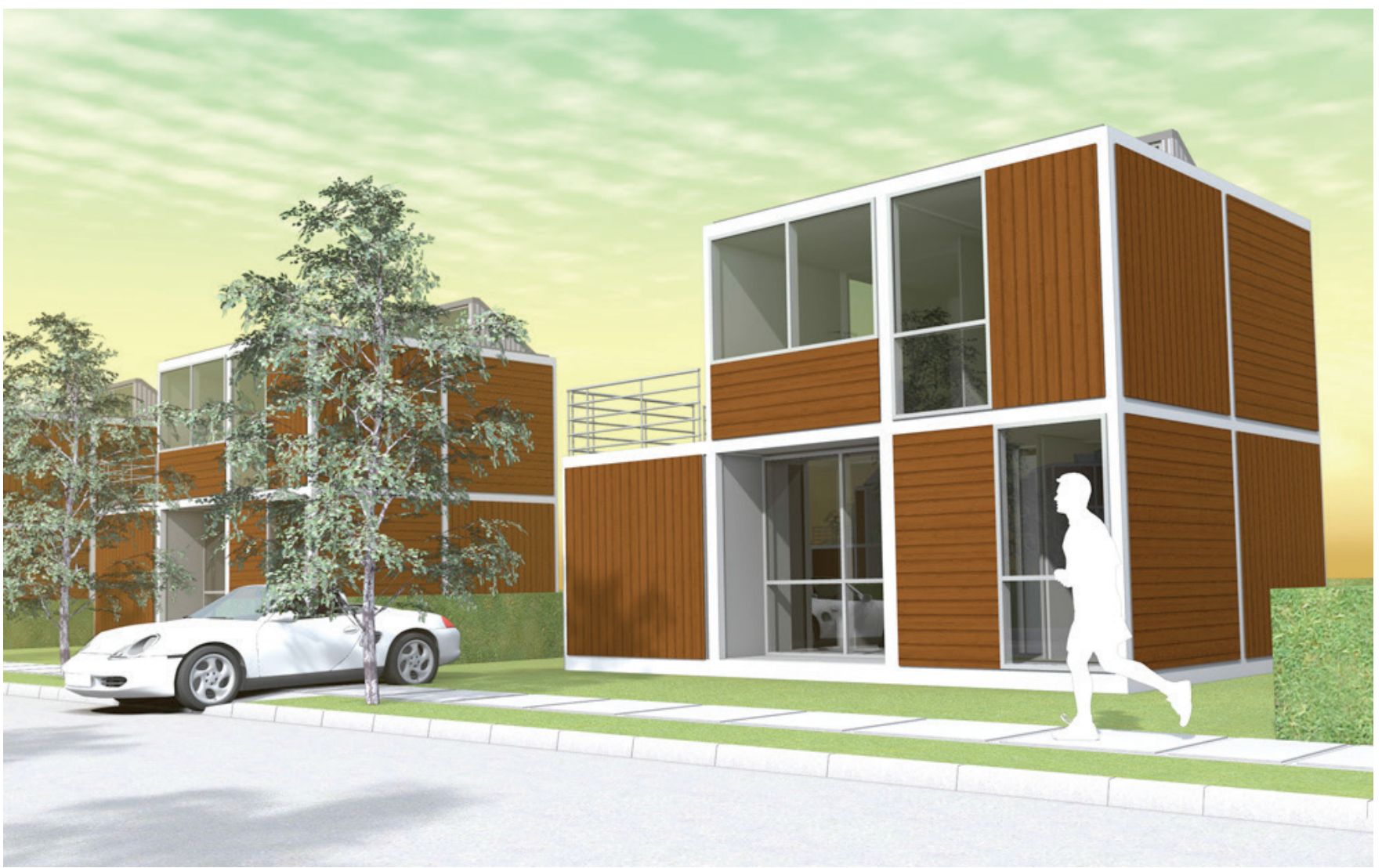

Figura 1: Vista de Viviendas Iniciales en el Sistema Casa+ 


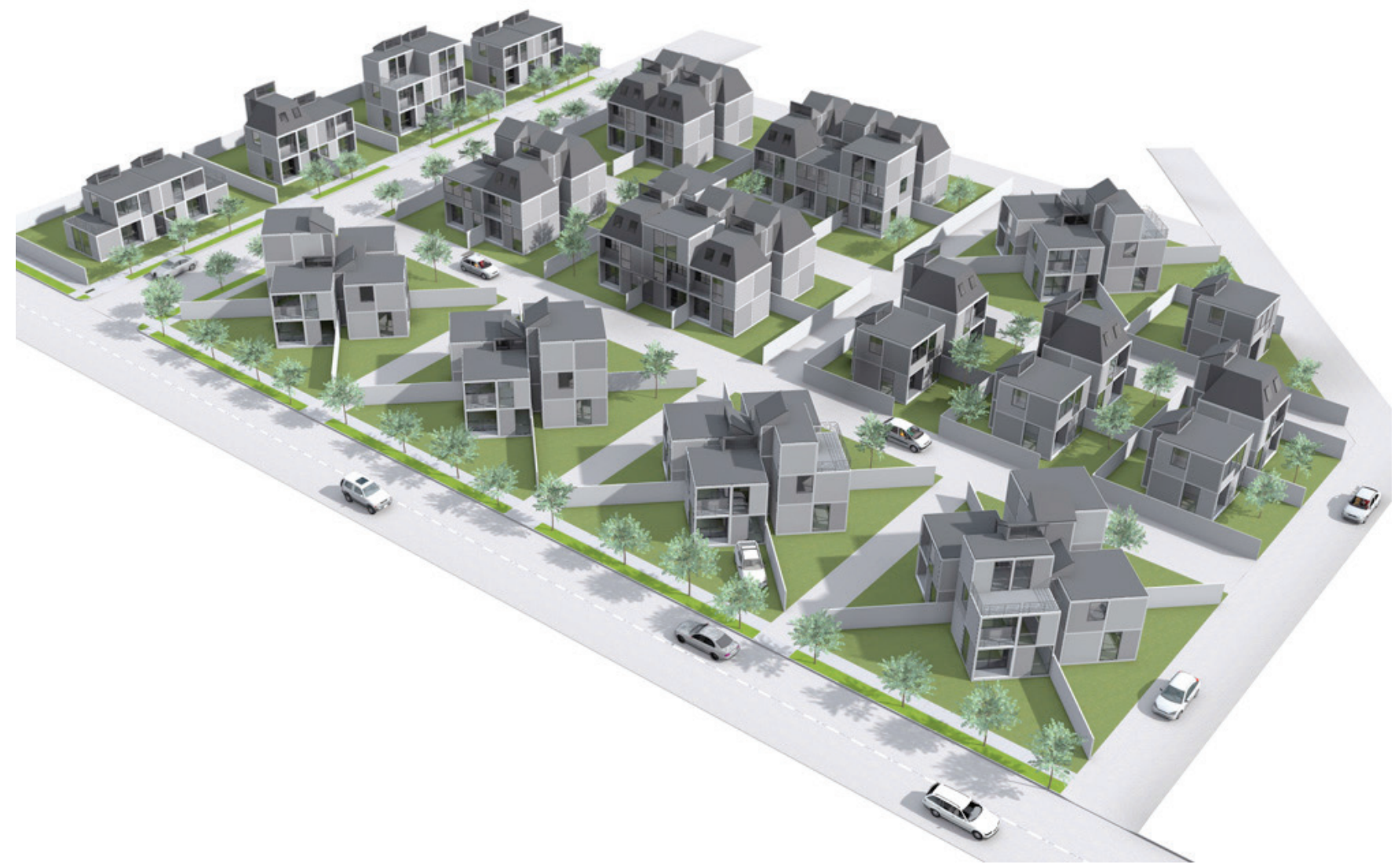

Figura 2: Vista con diferentes distribuciones iniciales en el Sistema Casa+

\section{Sistema Habitacional}

La propuesta desarrollada (Fig.1) consiste en un sistema prefabricado en estructura metálica que permiten componer distintas soluciones residenciales. Permitiendo un fabricación industrializada de rápida instalación y crecimiento progre-sivo, basado en una retícula tridimensional de 2,67 mts. de lado. La unidad habitacional básica se compone de dos módulos por lado y altura, más una caja de escala lateral de medio módulo, conformando $28,5 \mathrm{~m} 2$ en primer nivel y $57 \mathrm{~m} 2$ totales en dos niveles. La configuración inicial (o en sucesivas ampliaciones) puede alcanzar hasta ocho módulos en planta por lado y tres módulos de alto, por tanto hasta $228 \mathrm{~m} 2$, incluyendo también balcones o terrazas.

Los paramentos consideran paneles de tableros de fibra de madera dobles (SIP), con aislamiento interior orgánico de $100 \mathrm{~mm}$. a $150 \mathrm{~mm}$., con instalaciones, puertas y ventanas (con vidrios dobles) empotradas. Alcanzando transmitancias térmcas de 0,37 Wm2K en muros, $0,31 \mathrm{WM} 2 \mathrm{~K}$ en techos y $2,41 \mathrm{Wm} 2 \mathrm{~K}$ en ventanas. Con revestimientos exteriores hidrófugos (fibrocemento texturado) e interiores ignífugos (yeso-cartón), y barreras permeables de vapor.

En la organización interior se contemplan circulaciones en el costado posterior, con núcleo sanitario central y lugares habitables hacia el frente, con variaciones de disposición y aberturas mayores para asoleamiento (y protección superior para radiación directa de verano). Esto permite adosamiento posterior o lateral, en algunos casos desplazando el semi-módulo de escalera. Conformando conjuntos unidades continuas (con tres adosamientos por lado), que pueden ampliarse frontalmente y hacia arriba, alcanzando densidades residenciales sobre los 300 hab./ha. Hasta disposiciones más abiertas (Fig.2), adosando dos o cuatro unidades, rectas o en diagonal, lo que permite mayores ampliaciones (hasta cuadriplicar la superficie inicial), conservando eficiencia energética, captación solar y una densidad urbana cercana a los 150 hab./ha.

Para apoyar el calentamiento de agua se contempla inicialmente un panel solar de $4 \mathrm{~m} 2$ por unidad, soportado en las orientaciones preferentes por tragaluz en la escalera, que reduce consumo de iluminación. Para calefacción se considera un equipo a pellets de alta efectividad calorífica con mínima contaminación interior y huella ecológica. Para reducir infiltraciones se considera un sellado perimetral adicional, ventanas oscilo-batientes de ejecución controlada, rebordes de puertas y ventilación natural cruzada. Utilizando un intercambiador entálpico para ventilación forzada y recuperación de calor. 


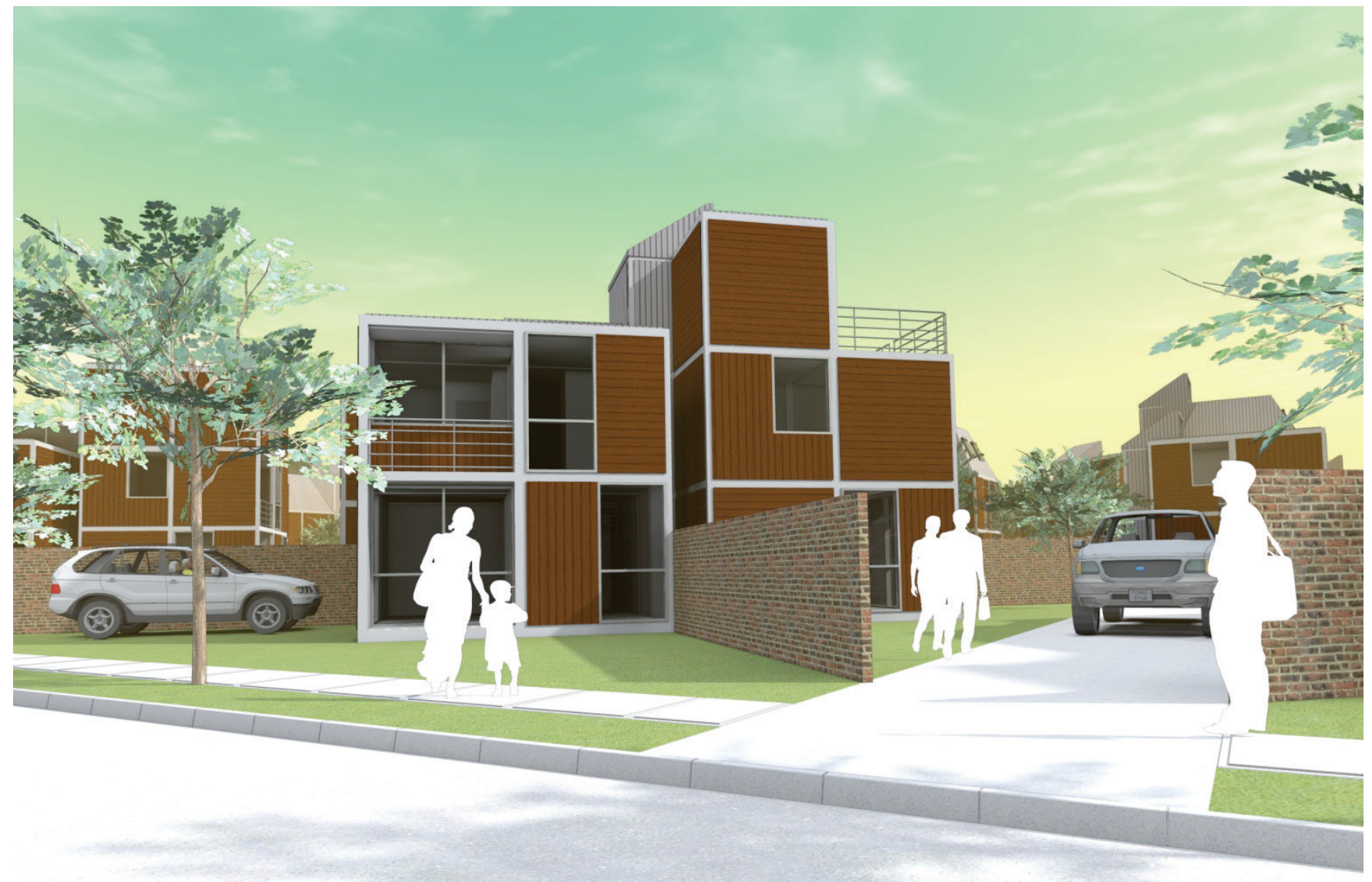

Figura 3: Vista de Viviendas Medias en el Sistema Casa+.

\section{Simulaciones}

Las simulaciones energéticas en régimen dinámico han demostrado la capacidad de predecir comportamientos ambientales y sugerir mejoramientos residenciales, en varias experiencias en la zona (Muller, 200; Trebilcock, 2001; Vera, 2002, Bustamante, 2009; Carrasco y Kokogiannakis, 2012). Sin embargo no se ha revisado su integración en el proceso de diseño, en relación con diferentes alternativas de configuración constructiva, arquitectónica y urbana, como se plantea en este trabajo. Seleccionando algunas condiciones relevantes, como la definición volumétrica, envolvente, orientación, ocupación y equipamiento de la vivienda.

Para analizar diferentes posibilidades de diseño y evaluar prestaciones estimadas se realizaron durante el desarrollo colaborativo del proyecto simulaciones ambientales en dos software (Ecotect y Casanova) para comparar resultados. Basados en el modelo BIM (Revit) de los módulos habitacionales básicos. Según antecedentes registrados, se consideró rango de confort: $18^{\circ}-27^{\circ} \mathrm{C}$, calefacción en régimen continuo $18^{\circ} \mathrm{C}$, ocupación 20 a 9 hrs (fin de semana completo), $12,5 \mathrm{~m} 2$ por persona, con ganancias internas de $5 \mathrm{kw}$ sensible y 2 kw latente, renovación de aire con infiltración de 1 ach. Obteniendo resultados de demanda energética globales y estimados por superficie (Tabla 3).

Tabla 3: DEMANDA TOTAL DE ENERGÍA Kwh/m2

\begin{tabular}{|l|c|c|c|}
\hline & ECOTEC & CASANOVA & \% Mejora \\
\hline $\begin{array}{l}\text { CASA + } \\
\text { SEGÚN RT }\end{array}$ & 160 & 140 & \\
\hline $\begin{array}{l}\text { CASA + } \\
\text { AISLADA }\end{array}$ & 47 & 43 & $70-75 \%$ \\
\hline $\begin{array}{l}\text { CASA+ } \\
\text { PAREADA }\end{array}$ & 38 & 36 & $75-80 \%$ \\
\hline $\begin{array}{l}\text { CASA+ } \\
\text { ADOSADA }\end{array}$ & 35 & 29 & $80-85 \%$ \\
\hline
\end{tabular}

La aplicación de software de simulación básico (Casanova) permite analizar rápidamente formas generales de la vivienda y configuración urbana. En programas más avanzados (Ecotect) se pueden revisar configuraciones materiales, comparando además costos y terminaciones en el grupo de trabajo (Crawley et al, 2008; Attia et al, 2009). El desempeño de sistemas interiores y equipos 
de energía renovable se analizó con procedimientos específicos. Además se elaboraron maquetas y estudios fotográficos para revisar los comportamientos lumínicos y apariencias. En la plataforma de modelación constructiva se desarrollaron también diferentes disposiciones de conjunto para revisar densidades, trazados viales y percepción de espacios públicos.

\section{Resultados}

En comparación con las condiciones regulares de las viviendas en la zona, el proyecto "Casat" presenta diferencias sustanciales (Tabla 4), especialmente en sus desempeños ambientales $y$ urbanos.

Tabla 4: Comparación de Resultados

\begin{tabular}{|l|l|l|}
\hline & $\begin{array}{l}\text { Vivienda } \\
\text { Convencional en la } \\
\text { Zona* }\end{array}$ & $\begin{array}{l}\text { Viv. Media de } \\
\text { Sistema Casa + }\end{array}$ \\
\hline $\begin{array}{l}\text { Confort Higrotérmico } \\
\text { (95\% del tiempo) }\end{array}$ & $13^{\circ}$ a $29^{\circ}$ con $85 \% \mathrm{HR}$ & $\begin{array}{l}20^{\circ} \text { a } 27^{\circ} \text { con } \\
75 \% \mathrm{HR}\end{array}$ \\
\hline $\begin{array}{l}\text { Calidad del Aire(95\% del } \\
\text { tiempo) }\end{array}$ & $\begin{array}{l}+1000 \mathrm{ppmC02} \\
2,5 \mathrm{ACH}\end{array}$ & $\begin{array}{l}-1000 \mathrm{ppmC02} \\
1,0 \mathrm{ACH}\end{array}$ \\
\hline Demanda Energética & $150 \mathrm{kWha} / \mathrm{m} 2$ & $30 \mathrm{kWha} / \mathrm{m} 2$ \\
\hline Aporte C02 & $70 \mathrm{ton} . \mathrm{a} / \mathrm{m} 2$ & 15 ton.a/m2 \\
\hline Operación & & \\
\hline Costo de Construcción & $15 \mathrm{UF} / \mathrm{m} 2$ & $12,96 \mathrm{UF} / \mathrm{m} 2$ \\
\hline Plazo de Ejecución & $4-5 \mathrm{meses}$ & $1-2 \mathrm{meses}$ \\
\hline Variedad de Tipologías & $\begin{array}{l}\text { Tres o Cuatro de } 70 \text { a } \\
100 \mathrm{~m} 2\end{array}$ & $\begin{array}{l}\text { Dieciocho de } \\
57 \text { a } 170 \mathrm{~m} 2\end{array}$ \\
\hline Crecimiento & No considerado & Planificado \\
\hline Densidad Urbana & $70 \mathrm{hab} . / \mathrm{ha}$ & $\begin{array}{l}150 \mathrm{a} \quad 300 \\
\text { hab./ha }\end{array}$ \\
\hline Distancia al centro & $10 \mathrm{~km}$. & $5 \mathrm{~km}$. \\
\hline
\end{tabular}

\section{Conclusiones}

Este trabajo demuestra que la aplicación de software de simulación energética y el trabajo colaborativo permite desarrollar proyectos habitacionales con mejoramientos sustanciales de densidad, programación constructiva, confort ambiental y reducción del consumo de combustibles. Aunque existen aprehensiones que el desarrollo colectivo y las evaluaciones técnicas, demoren o encarezcan el proceso de diseño, la experiencia demuestra que esto se incrementa escasamente con profesionales capacitados y motivados, obteniendo mejoramientos sustanciales del proyecto que justifican el esfuerzo.

Estos logros implican un trabajo arquitectónico con nuevas tecnologías de evaluación y modelación, así como compartir decisiones de diseño. Involucrando morfologías y estrategias constructivas innovadoras, enfocadas hacia prestaciones ambientales. Asegurando la calidad espacial y urbana. La propuesta de Casa+ comprueba las posibilidades de mitigar significativamente el impacto ecológico de las construcciones residenciales, otorgando calidad ambiental en conjuntos sociales participativos. Lo que sugiere nuevos caminos de desarrollo habitacional, como también enfoques profesionales y educacionales con mayor compromiso sostenible y aplicación de capacidades.

\section{Agradecimientos}

MEL-Conicyt 8110003 y Fondecyt 1120165.

\section{Referencias}

IEA (2012) "CO2 Emissions from Fuel Combustions Highlights", International Energy Agency: Paris.

CNE (2009), "Antecedentes sobre la matriz energética en Chile y sus desafíos futuros", Comisión Nacional de Energía: Santiago.

CDT, (2010), Estudio de Usos Finales y Curva de Oferta de Conservación de la Energía en el Sector Residencial. Cámara Chilena de la Construcción.

Celis, F.; Garcia, R.; Trebilcock. M.; Escorcia, O.; Bruscato U.; Diaz, M. (2012). Análisis energético de las viviendas del centro-sur de Chile. Arquiteturarevista (UNISINOS), v. 8, p. 62-75, 2012.

Bustamante W. (2009) Guía de Diseño para la Eficiencia Energética en la Vivienda. Social. Santiago, Ministerio de Vivienda y Urbanismo y programa País Eficiencia Energética.

Garcia R.; Bruscato U., Trebilcock, M; Celis, F ; Escorcia, O. (2012). Connecting up capacities:. Open House International, v. 37, p. 48-60, 2012.

Müller, E.: "Estudios Paramétricos con Simulaciones Térmicas para Viviendas con Climatización Pasiva en la Zona Central de Chile", en IX Congreso Chileno de Ingeniería Mecánica, Valparaíso, Chile, Octubre 10-14, 2000.

Trebilcock, M.; Burdiles, R.; \& Fissore, A.: "La modelación y simulación energético-ambiental como herramienta de rediseño arquitectónico", en Congreso Sigradi 2001, pp. 83-86. Concepción, Chile, Noviembre 21-23, 2001.

Vera, S.; Ordenes, M.: " Evaluación del Desempeño Energitérmico de una Vivienda Social en Chile, utilizando un programa de simulación energética de Edificios". Revista de la Construcción, vol 17 n³, pp. 133-142, 2002

Carrasco J. y Kokogiannakis, "Feasibility of PassivHaus standards and alternative passive design on climatic zones of Chile - Determination of energy requirements with dynamic simulation", Revista Habitat Sustentable, Vol.1, N², Concepción, 2012

Crawley D., Hand J., Kummert M y Grffith B. (2008) "Contrasting the capabilities of building energy performance simulation programs". Building and Environment, vol. 43 n4, 2008, p. 661-673.

Attia S., Beltran L., De Herde A. y Hensen . (2009), “Architect friendly: A comparison of ten different building performance simulation tools". En Actas de XI IBPSA Building Simulation Conference, vol. 49, p. 2-15 , 